\title{
In-plane Thermal Diffusivity Evaluation by Infrared Thermography
}

\author{
by F. Cernuschi ${ }^{1}$, A. Russo ${ }^{2}$, L. Lorenzoni ${ }^{1}$ \\ ${ }^{1}$ CESI Via Reggio Emilia, 39, 20090 Segrate (MI) Italy E-mail Cernuschi@cesi.it \\ 2 Politecnico di Milano, Dipartimento di Ingegneria Chimica e Chimica Industriale "G. Natta" \\ P.za Leonardo da Vinci 32, 20113 Milano Italy
}

\begin{abstract}
A new thermographic methodology for measuring the thermal diffusivity of a platelike sample is presented. In particular, the study of the time evolution of the spatial distribution of the surface temperature of the rear face of the plate (after heating its front surface by a flash Gaussian shaped source) enables to determine the in-plane thermal diffusivity. This technique is applied to an AISI304 stainless steel plate and the results are compared with literature value and with the value obtained on the same material by using Thermal Wave Interferometry.
\end{abstract}

\section{Introduction}

In the last fifteen years, the progress in infrared technology as well as in electronics have allowed to apply infrared thermography to the thermal diffusivity evaluation starting from the experience matured in the frame of phothermal science.

The main advantage of thermographic methods is related, in principle, to the possibility of obtaining either time or space resolved analysis of the surface temperature of the whole sample. In this way, new approaches to thermal diffusivity evaluation become possible or at least less time consuming.

Thermographic methods have been successfully developed for measuring thermal diffusivity in bulk materials [1] and both isotropic [2,3] and anisotropic [4-7] plates; these techniques typically allow to measure also the in-plane thermal diffusivity that is usually more difficult to be measured by conventional photothermal methods like Laser Flash [8].

Usually the methods applied for determining the in-plane thermal diffusivity exploit the inplane heat diffusion subsequent to a non uniform heating of the sample surface that can be achieved typically either using a non uniform heating source (i.e. a spotwise or a linewise source) $[1,2,9,10]$ or masking in a suitable way part of the sample surface during heating by a uniform source [5-7].

Thermal diffusivity can be estimated by analysing the time evolution or the spatial distribution or the phase image (the latter for lock-in thermography) of the temperature of either the front or the rear sample surface.

Here a thermographic technique for the in-plane thermal diffusivity evaluation is presented: it consists in analysing the time evolution of the spatial distribution of the temperature of the rear surface of a platelike sample heated on its front surface by an instantaneous circular Gaussian source.

\section{Theory}

The solution describing the temperature distribution within an infinite platelike isotropic sample of thickness $L$ whose front face is instantaneously heated by a circular Gaussian source of radius $R$ (defined as the distance where the beam intensity reduces by a factor $\left.1 / \mathrm{e}^{2}\right)$ if heat losses are neglected is: 


$$
T(r, z, t)=\frac{Q}{2 \varepsilon \sqrt{\pi^{3} t}} \sum_{n=-\infty}^{\infty} e^{-\frac{(n L-z / 2)^{2}}{\alpha t}} \int_{0}^{+\infty} \sigma J_{0}(\sigma r) e^{-\sigma^{2}\left(\frac{R^{2}}{8}+\alpha t\right)} d \sigma
$$

where $\alpha$ is the thermal diffusivity, $\varepsilon=\sqrt{k \rho C}$ ( $k \rho$ and $C$ are the thermal conductivity, the density and the specific heat respectively) is the thermal effusivity, $J_{0}(\sigma r)$ is the zero order first type Bessel function, $\mathrm{Q}$ is the total amount of heating energy, $t$ is the time, $z$ and $r$ are the axial and radial co-ordinates respectively. In particular, if the rear surface is considered, taking into account that [11]:

$$
\int_{0}^{+\infty} \sigma J_{0}(\sigma r) e^{-a \sigma^{2}} d \sigma=\frac{1}{2 a} e^{-\frac{r^{2}}{4 a}}
$$

Eq.(1) can be simplified as follows:

$$
T(r, L, t)=\frac{2 Q}{\varepsilon \sqrt{\pi^{3} t}} \sum_{n=-\infty}^{\infty} e^{-\frac{((2 n-1) L)^{2}}{4 \alpha t}} \frac{1}{\left(R^{2}+8 \alpha t\right)} e^{-\frac{2 r^{2}}{R^{2}+8 \alpha t}}
$$

Fig. 1 shows the temperature as a function of time at different distances from the beam centre as described by Eq.(3) while Fig. 2 shows the rear surface temperature spatial distribution as a function of the distance $r$ at different times.

As far as the thermal diffusivity measurement is concerned, the proposed technique consists in best fitting, at some fixed times, the spatial profile of the temperature along a line passing through the centre of the heated spot on the rear sample surface by using a Gaussian function; in fact the shape of the temperature profile at any fixed time depends only from the Gaussian term in eq.(3) as clearly shown by Fig. 2. At each time $t$ the value of the parameter:

$$
b^{2}=R^{2}+8 \alpha t
$$

is thus obtained.

The thermal diffusivity value can be obtained by using eq.(4) from the knowledge of both the value of the beam radius and the time the spatial distribution refers to; this procedure can be repeated at some different times in order to obtain an averaged value for thermal diffusivity.

\section{Experimental}

The experimental set-up is shown in Fig. 3. A $1000 \mathrm{~W}$ continuous lamp (PSC 1000 ILC Technology Inc. CA) was used as heating source. The beam was focused on the sample surface using a suitable lens system. A photographic shutter was used for producing the heating flash. The infrared camera used for measuring the surface temperature of the sample was a Focal Plane Array (320x240pixel) CEDIP Jade system sensitive in the spectral range $8-10 \mu \mathrm{m}$ with a NETD of $30 \mathrm{mK}$ and able to acquire snapshot infrared images with a frequency rate ranging from 50 up to $1000 \mathrm{~Hz}$ as a function of the image dimension. The shape and dimension of the heating spot impinging on the sample surface were measured analysing the IR image produced by the heating beam on a very thin black sheet of paper. Specifically, the heating spot approximated very well a Gaussian distribution as shown in Fig. 4. The beam radius $(7.7 \mathrm{~mm})$ was determined from the experimental data through a least- 
square analysis of the spatial profile of the temperature. In particular the procedure consisted in the four steps:

- to consider the temperature profile along two mutually normal lines crossing the heating spot;

- to fit them by a Gaussian in order to obtain the co-ordinates of the centre of the spot;

- to make the two lines passing through the centre of the spot;

- to fit temperature profiles along these two lines by a Gaussian in order to obtain the beam radius along both normal directions.

In order to be allowed to neglect the finite duration effects of the flash according to Maillet [12] and Cielo [13] the following conditions should be satisfied:

$$
\frac{\alpha \tau}{L^{2}}<0.05 \quad \text { and observation times } t>10 \tau
$$

As the sample considered for this study was an AISI304 stainless steel plate $(1.7 \mathrm{~mm}$ thick) with thermal diffusivity literature value $0.0410^{-4} \mathrm{~m}^{2} / \mathrm{s}$ [14], the first condition is satisfied for heating time duration $\tau$ equal to or shorter than $33 \mathrm{~ms}$ while the second one for observation times longer than 330ms.

\section{Results and Discussion}

As the procedure for the thermal diffusivity evaluation requires the precise values of the times the spatial profiles refer to, and as no synchronisation between the photographic shutter and the infrared camera was available, the initial time $t=0$ (corresponding to the flash) was not clearly determined. In order to solve this problem the following iterative procedure was developed:

- in first approximation to consider as initial time $t_{0}$ the one corresponding to the first IR image where the heating spot is distinguishable;

- to extract temperature spatial profiles at different times after $t_{0}$ and fit them by a Gaussian in order to obtain the parameter $b$;

- $\quad$ to determine the thermal diffusivity from eq.(3) ;

- to extract the temperature in the spot centre as a function of the time and to compare it with the one-dimensional model (Laser Flash); in particular in this way the correct time $t_{x}$ corresponding to a fixed percent rise $x$ (always lower than 10\%) of the temperature can be estimated by using the following equation [15]:

$$
t_{x}=\frac{k_{x} L^{2}}{\alpha}
$$

where $k_{\mathrm{x}}$ is a constant. After that, a new $t_{0}$ can be evaluated and the other times can be consequently corrected;

- $\quad$ to determine again the thermal diffusivity from eq.(3) using the corrected times.

This iterative process usually converges in very few steps and the approximation in using the one-dimensional model for temperature rise percentage lower than $10 \%$ affects the data in a negligible way. As a matter of fact the difference between the two curves at $10 \%$ is less than $5 \%$ which in this specific case corresponds to a time difference of $2 \mathrm{~ms}$. In Fig. 5 the experimental spatial profiles of temperature as well as the Gaussian curves are shown at two different times after the flash. The final thermal diffusivity was obtained averaging the values taken at 9 different times from 0.5 to $2.5 \mathrm{~s}$ and resulted $0.042 \pm 0.01 * 10^{-4} \mathrm{~m}^{2} / \mathrm{s}$.

Notwithstanding the satisfactory agreement between experimental and literature values [14], in order to get a further confirmation of the ability of this technique to estimate the thermal diffusivity, Thermal Wave Interferometry (TWI) [16] was also applied to a thin plate $660 \mu \mathrm{m}$ thick extracted from the platelike sample and a very good agreement between all the 
thermal diffusivity values was found. In fact the value obtained by TWI resulted $0.040 \pm 0.001 * 10^{-4} \mathrm{~m}^{2} / \mathrm{s}$.

In the theoretical model considered heat losses were completely neglected. As far as radiative heat losses are concerned, taking into account that the maximum temperature difference on the rear surface did not exceed $0.4^{\circ} \mathrm{C}$ their effect can be surely negligible.

Convective heat losses can be estimated considering the Biot number :

$$
B i=\frac{h d}{k}
$$

where $h, d$, and $k$ are the convection coefficient, a characteristic length and the material thermal conductivity respectively. In this case $d$ could be assumed as the length along which the temperature spatial profile is taken. For an AISI304 sample considering indoor free convective phenomena $(h=10 \mathrm{~W} / \mathrm{mK})$ and $d=6 R=6 * 10^{-2} \mathrm{~m}$, the Biot number results $(\cong 0.04)$ widely smaller than value $\mathrm{Bi}=0.1$ usually considered as the limit for neglecting convective heat losses.

\section{Bibliography}

[1] CERNUSCHI F., FABBRI L. and LAMPERTI M., "A new thermographic methodology for on-site thermal diffusivity determination" in AIP Conference Proceedings $\mathrm{n}^{\circ} 463$ Photoacoustic and Photothermal Phenomena, Rome, August 1998, edited by F. SCUDIERI and M. BERTOLOTTI, AIP Woodbury, New York,1999, p. 392-394.

[2] WELCH C. S., HEALTH D.M., and WINFREE W. P., "Remote measurement of in-plane diffusivity components in plates" J. Appl. Phys. 61 (3), 1987,p. 895-898.

[3] KRAPEZ J.C., "Simultaneous measurement of in-plane and out-of-plane diffusivity by using a grid-like mask”, 5 AITA 1999 Venice 29-30 September 1999.

[4] PHILIPPI I., BATSALE J.C., MAILLET D., and DEGIOVANNI A., "Measurement of thermal diffusivities through processing of infrared images”, Rev. Sci. Instrum. 66,1995, p. 182-191.

[5] ZHOUNG OUYANG, FAVRO L.D., and THOMAS R.L. "Measurement of diffusivities using infrared imaging of thermally blurried shadows" in AIP Conference Proceedings $n^{\circ} 463$ Photoacoustic and Photothermal Phenomena, Rome, August 1998, edited by F. SCUDIERI and M. BERTOLOTTI, AIP Woodbury, New York,1999, p. 374-376.

[6] VISSER E.P., VERSTEEGEN E.H., and VAN ENCKEVORT W.J.P., "Measurement of thermal diffusion in thin films using a modulated laser technique: application to chemicalvapor deposited diamond films" J. Appl. Phys. 71 (7), 1992, p. 3238-3248.

[7] KRAPEZ J.C., GARDETTE G., and BALAGEAS D., "Thermal ellipsometry in steady-state and by lock-in thermography: application to anisotropic materials characterization" in Proc. Quantitative Infrared Thermography QIRT '96 Eurotherm Seminar 50, September 2-5 1996, Stuttgart Germany, p. 257-261.

[8] PARKER W.P., JENKINS R.J., BUTTER C. P., GUTTER G. L. and ABBOTT G.L., "Flash method of determining thermal diffusivity, heat capacity, and thermal conductivity" J. Appl. Phys., 32, 1961, p.1679-1684.

[9] HE D., GU Y., ZHENG M. and ZHU D., "Measurement of thermal diffusivity of thin material using an infrared thermal wave imaging technique", $9^{\text {th }}$ Int. Topical Meeting on Photoacoustic and Photothermal Phenomena, Nanjing (China), 27-30 June 1996, S.Y. Zhang Ed., Progress in Natural Science, 1996, pp. 169-172.

[10] YAMANE T., KATAYAMA S., TODOKI M., "Measurement of thermal diffusivity parallel to the surface of platelike specimen by laser pulse heating method", Rev. Sci. Instrum. 67, (12) 1996, pp.4261-4267. 
[11] CARSLAW H.S. and JAEGER J.C., Conduction of heat in solids $2^{\text {nd }}$ Edition Clarendon Press Oxford 1959, p. $4909 \mathrm{n}^{\circ} 29$.

[12] MAILLET D., LACHI M., DEGIOVANNI A., "Simultaneous measurements of axial and radial thermal diffusivities of an anisotropic solid in thin plate: application to multilayered materials", Thermal Conductivity 21, Ed C.J. Cremers and H.A. Fine, Plenum Press, New York , 1990 p.90-107.

[13] BALAGEAS D., KRAPEZ J.C. and CIELO P., "Pulsed photothermal modeling of layered materials" J. Appl. Phys. 59, 1986 p.348-357.

[14] Web site:www.matls.com/.

[15] TAYLOR R.E., MAGLIC K.D., "Pulse method for thermal diffusivity measurement", Compendium of Thermophysical Property Measurement Methods Vol: 1: Survey of Measurement Techniques, Eds. K.D. Maglic et Al. Plenum Press New York 1984 p. 305-336.

[16] ALMOND D.P., PATEL P.M., Photothermal Science and Techniques, Chapman \& Hall, 1996, p.21-30.

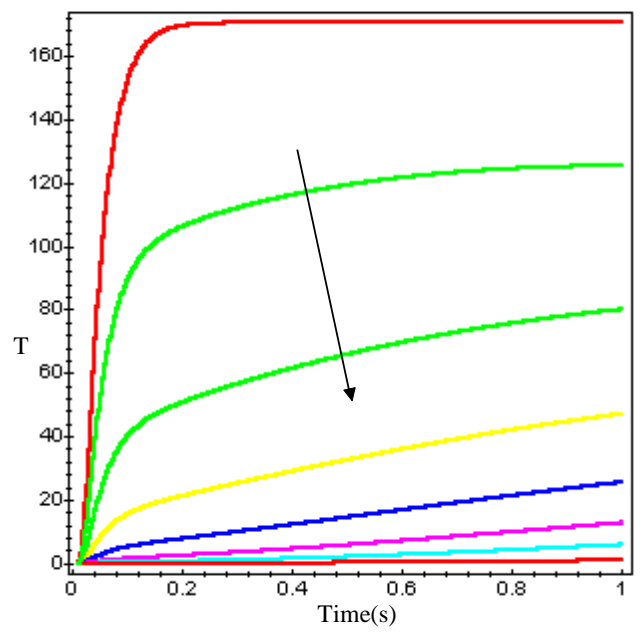

(a)

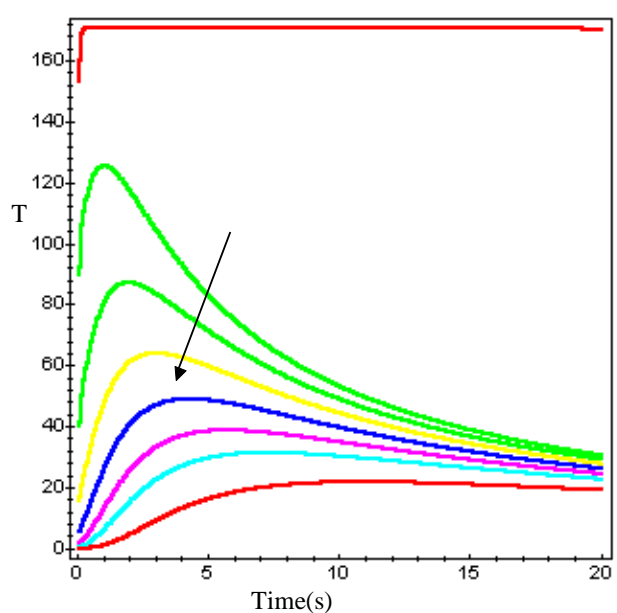

(b)

Fig. 1. Eq. (3). $T(r, L, t)$ as a function of the time. Curves refer to values of the distance $r$ from the Gaussian beam centre ranging from $5 \mathrm{~mm}$ to $12 \mathrm{~mm}$ increasing in the direction of the arrow. Computations have been performed fixing the beam radius $R=5 \mathrm{~mm}$ and slab thickness $1=1 \mathrm{~mm}$. (a) and (b) refer to two time ranges. In both (a) and (b), upper curve refers to the solution of the one dimensional problem.

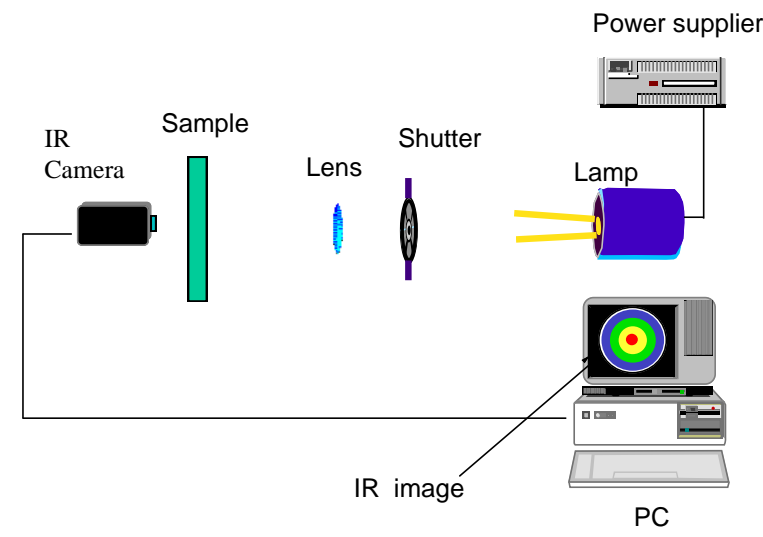

Fig. 3. Experimental set-up. 


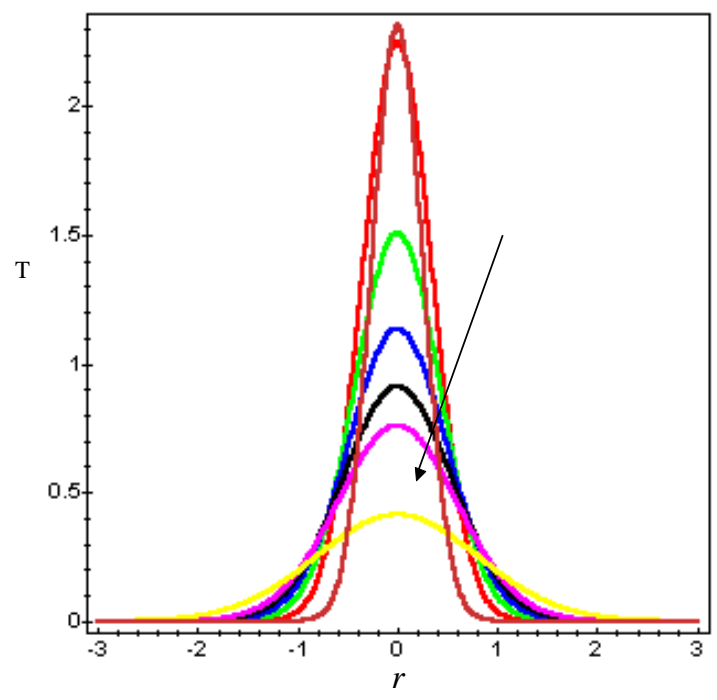

Fig. 2. Eq. (3). $T(r, L, t)$ as a function of the distance $r$. Curves refer to time values ranging from 1 to 10 seconds increasing in the direction of the arrow. Computations have been performed fixing the beam radius equal to $5 \mathrm{~mm}$ and slab thickness $1 \mathrm{~mm}$.

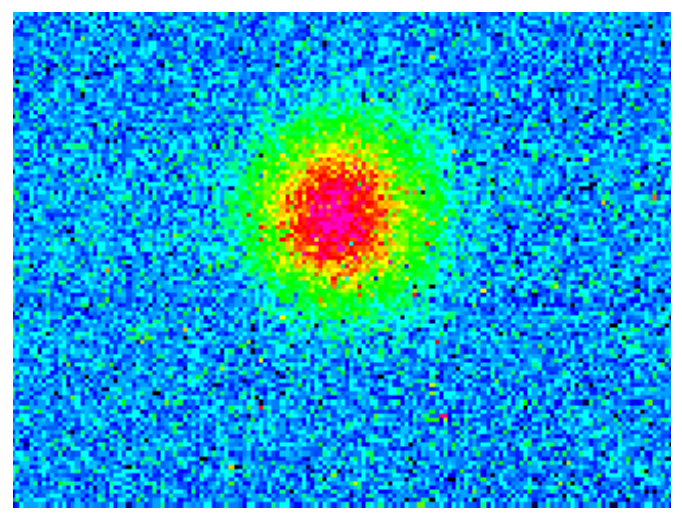

Fig. 4. IR image of the rear surface of the AISI304 stainless steel plate 1s after the flash heating.

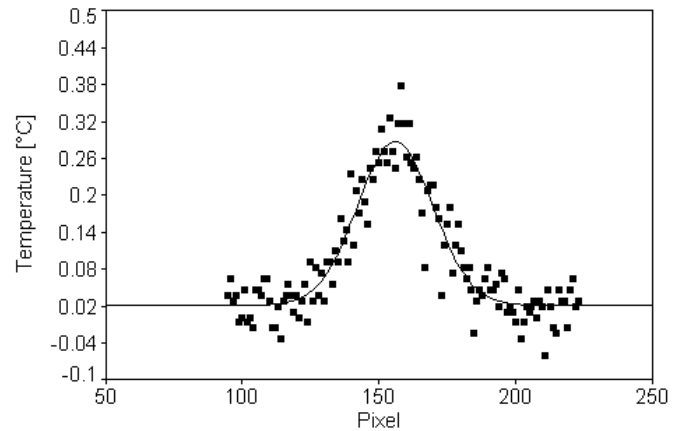

(a)

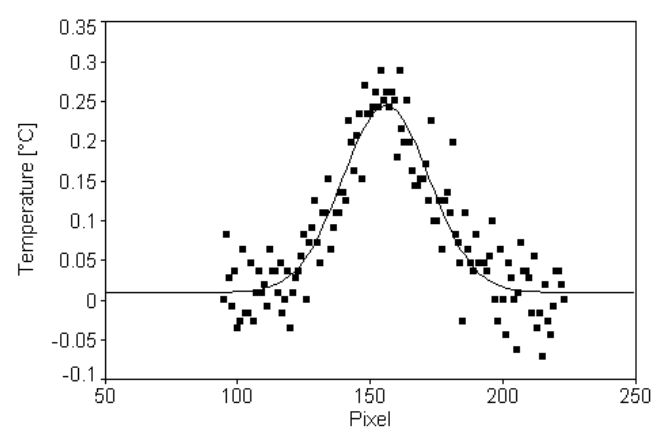

(b)

Fig. 5. Temperature versus spatial position at (a) $1 \mathrm{~s}$ and (b) $1.5 \mathrm{~s}$ after the heating pulse. 